\title{
Von Fall zu Fall
}

\author{
Rainer Sundmacher
}

Online publiziert: 14 . Juni 2017

(C) Springer-Verlag Wien 2017

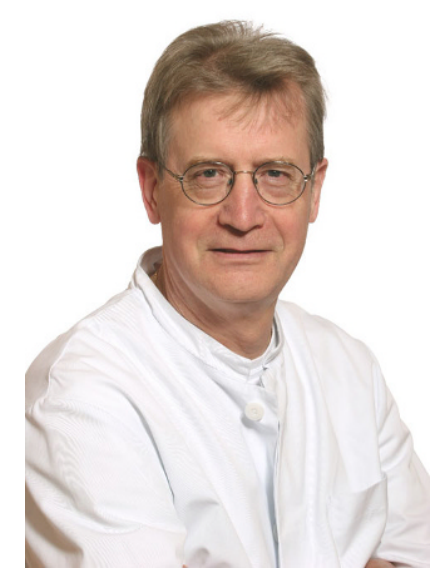

Die Arbeit Sundmacher R, Peter HH (1987) Cyclosporin A systemisch nach Keratoplastik wegen rheumatischer Hornhautperforation und bei essentieller Bindehautschrumpfung. Spektrum Augenheilk 1: 1-5 war meine 105. schriftliche Veröffentlichung von insgesamt mehr als 400 und eigentlich keine von denen, die meines Wissens sehr beachtet wurden. Für mich war sie aber wichtig und unverzichtbarer Ausgangspunkt vieler Arbeiten in den Jahrzehnten danach.

Als ich $1986 \mathrm{zu}$ einem Beitrag im ersten Heft des spektrum der augenheilkunde aufgefordert wurde, befand ich mich kurz vor meinem Wechsel von Freiburg nach Düsseldorf, um dort das Ordinariat von Hans Pau zu übernehmen und hatte so viel um die Ohren, wie man so sagt, dass nur die Einreichung einer kasuistischen Arbeit infrage kam, die aber einen anspruchsvollen Hintergrund haben und zugleich Entwicklungspotenzen für die Zukunft aufzeigen sollte.

Prof. Dr. R. Sundmacher $(\varangle)$

Schöneckstraße 5B, 79104 Freiburg, Deutschland r-sundmacher@t-online.de
Diesem Anspruch wird die Publikation aus meiner Sicht auch heute noch gerecht.

Ich hatte damals begonnen, systematisch nach Verbesserungsmöglichkeiten bei den Hochrisikokeratoplastiken zu suchen, z. B. nach À-chaud-Operationen bei Herpes sowie bei bakteriellen, mykotischen oder nichtinfektiösen („rheumatischen“) Hornhautperforationen sowie bei essenziellen Bindehautschrumpfungen. Der Fokus lag damals fast ausschließlich auf einer besseren Beherrschung der immunologischen Transplantatabstoßungen, von der wir uns große Fortschritte erhofften.

Die dargestellten 2 Fälle ergaben ein sehr unterschiedliches Bild. Zum einen erlaubten sie vorherzusagen, dass sich unsere Hoffnung mithilfe des damals erstmals in die klinische Praxis gelangenden Cyclosporin A als neuem Immunmodulativum wahrscheinlich erfüllen würde (z. B. bei den „rheumatischen“, immunologisch determinierten Hornhautperforationen). Zum anderen würden Verbesserungen der Immunmodulation aber vermutlich leider auch wirkungslos bleiben wie bei der essenziellen Bindehautschrumpfung, bei der - ungeachtet der Effizienz einer Immunmodulation - offenbar andere Faktoren das Überleben eines Hornhauttransplantates in erster Linie bestimmten.

Wer sich also in den Grundproblematiken der Keratoplastik ein wenig auskannte, konnte anhand der beiden Kasuistiken schon ziemlich sicher vorhersehen, „wohin die Reise gehen würde“: Die damals von mir in der deutschsprachigen Literatur im spektrum der augenheilkunde erstmals thematisierte systemische Cyclosporin-A-Immunmodulation bei Keratoplastik sollte sich in den Jahrzehnten danach als fester Therapiebestandteil zur Verhinderung der immunologischen Abstoßungen bei einer Fülle von Hochrisikokeratoplastiken erweisen, sogar beim bis dahin überhaupt 
nicht operierbaren Ulkus Mooren, wie wir später zeigen konnten.

Einem Erkrankungskomplex aber blieben bis heute die Segnungen einer optimierten Immunmodulation vorenthalten, und das sind all jene Erkrankungen, die mit einer dauerhaften Zerstörung des HornhautBindehaut-Epithels einhergehen, letztlich also mit einem irreparablen vollständigen Untergang der Limbusstammzellen, sei er nun endogen (z. B. essenzielle Bindehautschrumpfung) oder exogen herbeigeführt (z.B. schwerste Verbrennungen, Verätzungen oder Bestrahlungsschäden). Wenn dieses System völlig verschwunden und die Augenoberfläche vernarbt ist, hilft, wie wir erfahren mussten, auch keine vorherige Transplantation von homologen Limbusstammzellen mehr, weil diese - so unsere Vermutung - kein Gewebemilieu mehr vorfinden, in dem sie überleben können. Sie sterben über kurz oder lang ab, und nachfolgende Hornhauttransplantationen sind gänzlich erfolglos und hoch gefährlich, weil sie im schlimmsten Fall den Verlust des Augapfels bedeuten.

Ich möchte also urteilen: Alles ist bis heute richtig, was vor 30 Jahren geschrieben wurde, auch wenn die pathophysiologischen Hintergründe noch nicht so klar waren, wie sie heute vielen erscheinen - ich sage bewusst „erscheinen“, weil sie im Detail tatsächlich auch heute noch reichlich unklar sind.

Eines möchte ich abschließend aber noch tun: eine prinzipielle Lanze für die Publikation sorgfältig beobachteter und analysierter Einzelfälle brechen. In unserer heutigen Publikationswelt bestimmt fast nur noch der sog. „Evidenzlevel“ den Öffentlichkeitswert von wissenschaftlichen klinischen Arbeiten. Den höchsten haben doppelblind randomisierte Studien, den geringsten Fallbeschreibungen. Das hat natürlich seine objektive Berechtigung, führt aber leicht dazu, dass Nachwuchswissenschaftler sich scheuen, Fallbeschreibungen überhaupt zur Publikation einzureichen, weil sie um ihren „Ruf“ als solide Wissenschaftler fürchten; und die Gutachter "renommierter“ internationaler Zeitschriften weisen Fallbeschreibungen heutzutage oft schon grundsätzlich $a b$, ohne deren potenzielle Wichtigkeit überhaupt abzuschätzen. Es wird dabei geflissentlich außer Acht gelassen, dass die sorgfältige Einzelfallbeobachtung und ihre Publikation unverzichtbare Basis jeden wissenschaftlichen Fortschritts in der klinischen Medizin sind. Erst nachdem man Einzelfälle sorgfältig beobachtet, analysiert und dann weitergehend spekuliert hat, was das, was man beobachtet hat, vermutlich bedeutet, dann erst kann man zu „höheren“ und durchaus notwendigen „beweisenden“ Formen der klinischen Studien weiterschreiten. Dass wir uns zu Beginn allen Fortschritts „von Fall zu Fall“ forthangeln müssen, daran kommen wir aber nicht vorbei, und zu loben sind alle Redaktionen, die diesem Faktum mit Aufgeschlossenheit entsprechen wie damals die Redaktion des spektrum der augenheilkunde.

Interessenkonflikt R. Sundmacher gibt an, dass kein Interessenkonflikt besteht. 